\title{
Hydrodynamics beyond Navier-Stokes: Exact Solution to the Lattice Boltzmann Hierarchy
}

\author{
S. Ansumali \\ School of Chemical and Biomedical Engineering, Nanyang Technological University, 639798 Singapore, Singapore \\ I. V. Karlin* \\ Aerothermochemistry and Combustion Systems Lab, ETH Zurich, 8092 Zurich, Switzerland \\ S. Arcidiacono \\ Paul Scherrer Institute, Combustion Research, 5232 Villigen PSI, Switzerland \\ A. Abbas \\ School of Chemical and Biomedical Engineering, Nanyang Technological University, 639798 Singapore, Singapore \\ N. I. Prasianakis \\ Aerothermochemistry and Combustion Systems Lab, ETH Zurich, CH-8092 Zurich, Switzerland
}

(Received 26 July 2006; published 22 March 2007)

\begin{abstract}
The exact solution to the hierarchy of nonlinear lattice Boltzmann (LB) kinetic equations in the stationary planar Couette flow is found at nonvanishing Knudsen numbers. A new method of solving LB kinetic equations which combines the method of moments with boundary conditions for populations enables us to derive closed-form solutions for all higher-order moments. A convergence of results suggests that the LB hierarchy with larger velocity sets is the novel way to approximate kinetic theory.
\end{abstract}

DOI: 10.1103/PhysRevLett.98.124502

PACS numbers: 47.11.- j, 05.70.Ln

The emerging field of fluid dynamics at the micrometer scale becomes increasingly important due to fundamental engineering issues of micro-electromechanical systems [1]. Recently, much attention was focused on the use of lattice Boltzmann (LB) models for simulation of microflows by a number of groups [2-5]. By now, it is understood that lattice Boltzmann models form a well-defined hierarchy based on discrete velocity sets with velocities defined as zeros of Hermite polynomials [6] or rationalnumber approximations thereof [7]. The LB hierarchy constitutes a novel approximation of the Boltzmann equation and has to be considered as an alternative to more standard approaches such as higher-order hydrodynamics (Burnett or super-Burnett) or Grad's moment systems (for a review see, e.g., [8]). One salient feature of the LB hierarchy, which is crucial to the present study and eventually to any realistic application, is that it is naturally equipped with relevant boundary conditions derived from Maxwell-Boltzmann theory [2].

Agreement between LB simulations and kinetic theory [2], hydrodynamics with slip boundary conditions [5], and molecular dynamics was reported. However, most of these numerical works rely on simulation with finite accuracy while the crucial question of whether or not the kinetic equations underpinning the LB method are valid physical models of microflow remains unanswered. Therefore, it is not surprising to read comments claiming, for example, that the slip flow in the LB method is due to discretization errors rather than a physical effect, ([9] and references therein). Thus, validity of LB method cannot be addressed unless a comparison to representative exact solutions is performed. It is needless to say that exact solutions to nonlinear kinetic equations in realistic geometries are very rare.

In this Letter, we show that the LB hierarchy of kinetic models admits a much more accurate treatment. In particular, we find closed-form analytical solutions to nonlinear kinetic equations of the LB hierarchy in the stationary planar Couette flow. Not only the slip velocity, but also the shear stress and normal stress difference are evaluated in a closed form. Comparison to the kinetic theory demonstrates convergence of approximations with the increase of the number of velocities. In the nonlinear domain, even the first member of the LB hierarchy predicts nontrivial normal stress which is confirmed with a more microscopic direct simulation Monte Carlo (DSMC) method [10]. The accurate results obtained herein strongly suggest that the LB hierarchy should be considered as a novel general tool of kinetic theory rather than a plain solver for hydrodynamics.

Kinetic equations studied in this Letter are twodimensional isothermal discrete velocity models with the Bhatnagar-Gross-Krook (BGK) nonlinear collision integral (for a derivation of these models from the Boltzmann-BGK equation see, e.g., [6,7]),

$$
\partial_{t} f_{i}+c_{i \alpha} \partial_{\alpha} f_{i}=-\frac{1}{\tau}\left(f_{i}-f_{i}^{\mathrm{eq}}\right),
$$

where summation convention is applied, $\tau$ is the relaxation time, and the equilibrium distribution is 


$$
f_{i}^{\mathrm{eq}}=w_{i}\left(\rho+\frac{j_{\alpha} c_{i \alpha}}{c_{s}^{2}}+\frac{j_{\alpha} j_{\beta}}{2 \rho c_{s}^{4}}\left(c_{i \alpha} c_{i \beta}-c_{s}^{2} \delta_{\alpha \beta}\right)\right) .
$$

Here $\rho=\sum_{i=0}^{8} f_{i}$ is the density, $j_{\alpha}=\sum_{i=0}^{8} c_{i \alpha} f_{i}$ is the momentum density, $c_{s}=\sqrt{\left(k_{B} T_{0}\right) / m}$ is the speed of sound, $T_{0}$ is the reference temperature, and $m$ is particle's mass. The first member of the LB hierarchy is the so-called $D 2 Q 9$ model where the discrete velocities $c_{i \alpha}, i=$ $0, \ldots, 8$, and the weights $w_{i}$ are

$$
\begin{aligned}
c_{x} & =\sqrt{3} c_{s}\{0,1,0,-1,0,1,-1,-1,1\}, \\
c_{y} & =\sqrt{3} c_{s}\{0,0,1,0,-1,1,1,-1,-1\}, \\
w & =(1 / 36)\{16,4,4,4,4,1,1,1,1\} .
\end{aligned}
$$

The model (1) conserves density and momentum density but not energy. In the hydrodynamic limit, it recovers the Navier-Stokes equations with the kinematic viscosity $\nu=$ $\tau c_{s}^{2}$.

We consider the planar Couette flow, where a fluid is enclosed between two parallel plates separated by a distance $L$. The bottom plate at $y=-L / 2$ moves with the velocity $U_{1}$ and top plate at $y=L / 2$ moves with the velocity $U_{2}$. Let us introduce the mean free path $l=$ $\sqrt{3} \tau c_{s}$ and the Knudsen number $\mathrm{Kn}=l / L$. The solution for the $x$ velocity of the $D 2 Q 9$ model derived below reads

$$
u_{x}=\frac{1}{\Theta_{9}}\left(\frac{y}{L}\right) \Delta U+U,
$$

where $\Theta_{9}=1+2 \mathrm{Kn}$, and where $\Delta U=U_{2}-U_{1}$ is the relative velocity of the plates, and $U=\left(U_{1}+U_{2}\right) / 2$ is the centerline velocity. Solution (4) is Galilean invariant. The slip velocity at the plates, $u_{x}( \pm L / 2)$, features the expected behavior from a linear increase with $\mathrm{Kn}$ at small $\mathrm{Kn}$ to a plug flow at $\mathrm{Kn} \rightarrow \infty$ where $u_{x}$ becomes position independent. Recent careful numerical study of the $D 2 Q 9$ model by Sofonea and Sekerka [5] revealed the same result (4).

The next member of the LB hierarchy is the model based on the roots of fourth-order Hermite polynomial $\{ \pm a, \pm b\}$, where $a=\sqrt{3-\sqrt{6}}$ and $b=\sqrt{3+\sqrt{6}}$. In two dimensions, the discrete velocities are all possible tensor products of the two copies of the four sets $\{ \pm a, \pm b\}$. For this $D 2 Q 16$ model [6,7], the solution for the velocity profile is found to be

$$
u_{x}=\frac{1}{Z_{16}} \sinh \left(\frac{y}{\mathrm{Kn} L}\right) \Delta U+\frac{1}{\Theta_{16}}\left(\frac{y}{L}\right) \Delta U+U,
$$

where

$$
\begin{aligned}
\Theta_{16}=1+2 \operatorname{Kn}\left[\frac{2 \cosh \left(\frac{1}{2 \mathrm{Kn}}\right)+\mu \sinh \left(\frac{1}{2 \mathrm{Kn}}\right)}{\mu \cosh \left(\frac{1}{2 \mathrm{Kn}}\right)+2 \sqrt{3} \sinh \left(\frac{1}{2 \mathrm{Kn}}\right)}\right], \\
Z_{16}=\frac{\mu}{4 \mathrm{Kn}}\left[(4 \mathrm{Kn}+\mu) \cosh \left(\frac{1}{2 \mathrm{Kn}}\right)\right. \\
\left.+2(\mu \mathrm{Kn}+\sqrt{3}) \sinh \left(\frac{1}{2 \mathrm{Kn}}\right)\right]
\end{aligned}
$$

and $\mu=a+b \approx 3.076$. The difference between (4) and (5) is that the latter predicts the boundary Knudsen layer [first term in (5)] in a qualitative agreement with kinetic theory [11]. On the quantitative side, our analytical results can be immediately compared with the classical study of the linearized Boltzmann-BGK equation by Willis [12] (see Fig. 1, where also the results of the DSMC simulation are reported; note that the data in Fig. 1 are parametrized with the Knudsen number according to a relation, $\mathrm{Kn}=$ $\sqrt{3 / 2} \alpha^{-1}$, where $\alpha=\frac{L}{\tau} \sqrt{m /\left(2 k_{B} T_{0}\right)}$ is a parameter used in Table I of Ref. [12]). While the simplest $D 2 Q 9$ model predicts well a slip-flow solution, it fails in the transient regime $(\mathrm{Kn} \gtrsim 0.1)$, in agreement with numerical studies [2]. However, already the $D 2 Q 16$ model considerably improves the situation. The strong pattern of convergence with increasing the number of velocities in the LB hierarchy is clearly there.

We shall now proceed with major steps of derivation for the $D 2 Q 9$ model. First, the kinetic equation for nine populations (1) is rewritten in a form of a moment system for nine moments which we choose as follows: three locally conserved fields, $\rho, j_{x}, j_{y}$; three independent components of the pressure tensor, $P_{\alpha \beta}=\sum_{i=0}^{8} f_{i} c_{i \alpha} c_{i \beta}$, which we choose as the trace $P=P_{x x}+P_{y y}, N=P_{x x}-P_{y y}$ (normal stress difference), and $P_{x y}$; two components of the energy flux, $q_{\alpha}=\sum_{i=0}^{8} f_{i} c_{\alpha i} c_{i}^{2}$, and a scalar fourth-order moment, $\psi=R_{y y y y}+R_{x x x x}-2 R_{x x y y}$, where $R_{\alpha \beta \gamma \theta}=$ $\sum_{i=0}^{8} f_{i} c_{i \alpha} c_{i \beta} c_{i \gamma} c_{i \theta}$. The moment system is easily obtained and is not displayed here.

Second, we find steady state solution to the moment system under two conditions: (i) unidirectional flow: as the plates extend to infinity in the $x$ direction, we can expect that the steady state solution will be independent of $x$, and (ii) impermeable plates: the normal mass flux equals zero at the walls. For a unidirectional stationary flow, balance equations of density and of the two components of momentum read $\partial_{y} j_{y}=0, \partial_{y} P_{x y}=0$, and $\partial_{y}(P-$ $N)=0$, whereupon, using condition (ii), we get $j_{y}=0$, $P_{x y}=P_{x y}^{\text {neq }}, P=N+P_{0}$, where integration constants $P_{x y}^{\text {neq }}$
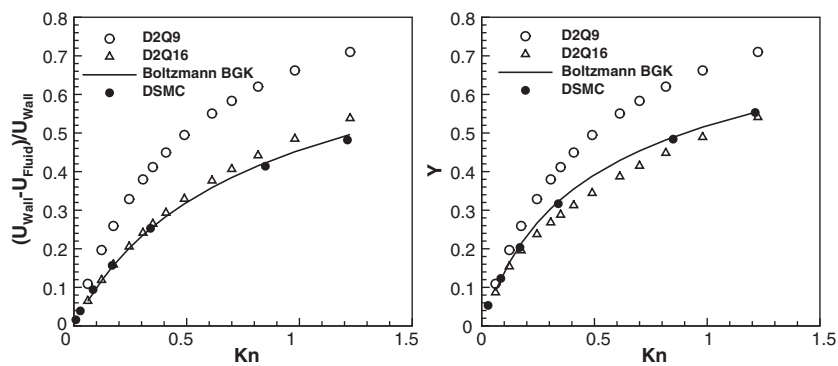

FIG. 1. Comparison of the LB hierarchy with the linearized Boltzmann-BGK model [12] and DSMC simulation. Left: Slip velocity at the wall as a function of Knudsen number. Right: Slope of the velocity profile at the centerline. Plotted is the deviation from the Navier-Stokes prediction, $Y=$ $\left.\Delta U^{-1}\left(d u_{x} / d y\right)\right|_{y=0}-1$. 

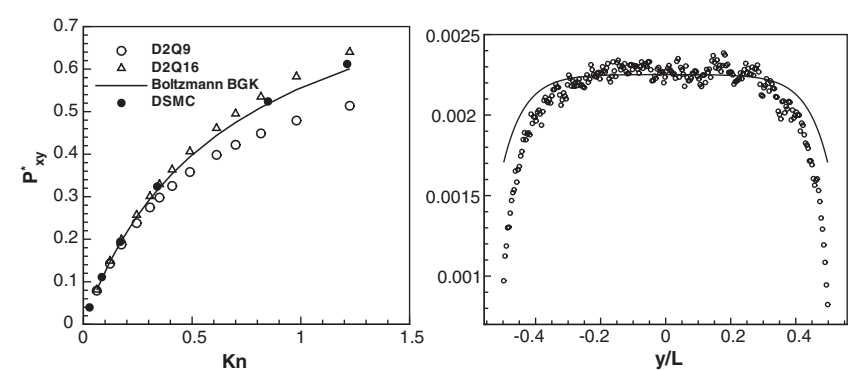

FIG. 2. Left: Shear stress at various Knudsen numbers. Labels are as in Fig. 1. Plotted is the reduced function $P_{x y}^{*}=P_{x y} / P_{x y}^{\infty}$ where $P_{x y}^{\infty}$ is the shear stress at $\mathrm{Kn} \rightarrow \infty$ of the Boltzmann-BGK model [12]. Right: Nonequilibrium normal stress difference at $\mathrm{Kn}=0.6$. Line: solution (21); symbol: DSMC simulation.

and $P_{0}$ will be determined below (superscript "neq" is added to emphasize that only the nonequilibrium part is nontrivial for $P_{x y}$ ). Furthermore, equation for the pressure tensor reads

$$
\begin{gathered}
\partial_{y}\left(q_{x}-3 c_{s}^{2} j_{x}\right)=-\frac{1}{\tau} P_{x y}^{\mathrm{neq}}, \\
\partial_{y} q_{y}=\frac{1}{\tau}\left(\frac{j_{x}^{2}}{\rho}-N\right), \\
\partial_{y} q_{y}=\frac{1}{\tau}\left(2 \rho c_{s}^{2}+\frac{j_{x}^{2}}{\rho}-N-P_{0}\right) .
\end{gathered}
$$

From (9) and (10) it follows $P_{0}=2 \rho c_{s}^{2}$. Thus, the stationary density is constant. Equation for energy flux reads

$$
\begin{gathered}
q_{x}=4 c_{s}^{2} j_{x}, \\
\partial_{y}\left[3 c_{s}^{2}\left(P-\frac{1}{2} N\right)-\frac{1}{2} \psi\right]=-\frac{1}{\tau} q_{y} .
\end{gathered}
$$

Substituting (11) into (8), and integrating the resulting differential equation for $j_{x}$, we obtain the result for the nontrivial velocity component $u_{x}=j_{x} / \rho$,

$$
u_{x}(y)=-\frac{\pi}{\tau c_{s}^{2}}(y+\tau V),
$$

where $V$ is constant of integration, and where we have introduced $\pi=P_{x y}^{\text {neq }} / \rho$. Thus, we find the solution for the velocity (up to two constants, $\pi$ and $V$ ) before higher-order moments are addressed. We note in passing that it is precisely the relation (11) pertinent to the low-symmetry $D 2 Q 9$ model (the energy flux is proportional to the momentum flux) which precludes the development of the boundary Knudsen layer. This constraint is removed in the more symmetric $D 2 Q 16$ model and in any higher-order member of the LB hierarchy.

The stationary equation for fourth-order moment $\psi$ together with (9), gives $\psi=4 c_{s}^{2} \frac{j_{x}^{2}}{\rho}-3 c_{s}^{2} N+4 \rho c_{s}^{4}$. Finally, from (9) and (12), we get

$$
\partial_{y} q_{y}^{\text {neq }}=-\frac{N^{\text {neq }}}{\tau}, \quad \partial_{y} N^{\text {neq }}=-\frac{q_{y}^{\text {neq }}}{3 \tau c_{s}^{2}}-\frac{\rho}{3} \partial_{y}\left(u_{x}^{2}\right) .
$$

The ordinary differential equations (14) can be integrated explicitly since the velocity $u_{x}(y)$ is available from (13). Denoting $\varphi(y)=\exp \left(y / \sqrt{3} \tau c_{s}\right)$, the result is

$$
\begin{aligned}
\sqrt{3} c_{s} N^{\text {neq }} \pm q_{y}^{\text {neq }}= & A_{ \pm} \varphi(\mp y) \mp \frac{2 \rho \pi^{2}}{\tau c_{s}^{2}} \\
& \times\left\{y+\tau\left(V \mp \sqrt{3} c_{s}\right)[1-\varphi(\mp y)]\right\},
\end{aligned}
$$

where $A_{+}$and $A_{-}$are constants of integration. Thus, the solution to the stationary moment system depends on the four integration constants, $\pi, V, A_{+}$, and $A_{-}$. To determine these, we need to specify boundary conditions at the moving plates. Note that this is precisely where the LB hierarchy differs from the method of moments. It is well known that for moment methods, such as Grad's systems, it is not possible to provide self-consistent boundary conditions for the moments. In our case, this is possible because the boundary conditions for the LB equations are formulated in terms of populations rather than in terms of moments. Upon inverting the linear relation between the moments and the populations, and using the solution for the moments derived above, we obtain the stationary populations $f_{i}=f_{i}^{\text {eq }}+f_{i}^{\text {neq }}$, where the stationary equilibrium part is given by (2) with $j_{y}=0$ and $j_{x}=\rho u_{x}$ (13), while the nonequilibrium part has the form

$$
\begin{aligned}
f_{i}^{\mathrm{neq}}= & w_{i}\left[\frac{P_{x y}^{\mathrm{neq}}}{c_{s}^{4}} c_{i x} c_{i y}+\frac{q_{y}^{\mathrm{neq}}}{2 c_{s}^{6}}\left(c_{i y} c_{i}^{2}-4 c_{s}^{2} c_{i y}\right)\right. \\
& \left.+\frac{N^{\text {neq }}}{2 c_{s}^{6}}\left(c_{i x}^{2}-c_{s}^{2}\right) c_{i y}^{2}\right] .
\end{aligned}
$$

Third and finally, we apply the classical diffuse boundary conditions [11], which were adapted to the present model in Ref. [2]. At the bottom plate $(y=-L / 2)$, diffuse boundary condition in the steady state is

$$
\left.f_{2,5,6}\right|_{y=-L / 2}=f_{2,5,6}^{\mathrm{eq}}\left(\rho, \rho U_{1}, 0\right) .
$$

In other words, in the steady state, the diffuse boundary condition reduces to setting the corresponding populations at equilibrium (2) with the density $\rho$ and velocity of the wall. Now, in order to find a relation between the two integration constants $V$ and $\pi$, we notice that the difference $\left[f_{5}^{\text {neq }}-f_{6}^{\text {neq }}\right]_{y=-L / 2}$ can be evaluated in two ways. On one hand, $\left[f_{i}^{\text {neq }}\right]_{y=-L / 2}=\left[f_{i}\right]_{y=-L / 2}-\left[f_{i}^{\mathrm{eq}}\right]_{y=-L / 2}$, where the first contribution is due to (17), and the second is due to the stationary solution for the equilibrium (2) with the velocity (13), whereupon $\left[f_{5}^{\text {neq }}-f_{6}^{\text {neq }}\right]_{y=-L / 2}=\left(\sqrt{3} \rho / 18 c_{s}\right)\left[U_{1}+\right.$ $\left.\frac{\pi}{\tau c_{s}^{2}}\left(-\frac{L}{2}+\tau V\right)\right]$. On other hand, using (16), we find $\left[f_{5}^{\text {neq }}-\right.$ $\left.f_{6}^{\text {neq }}\right]_{y=-L / 2}=\frac{\rho \pi}{6 c_{s}^{2}}$. Matching these expressions, we find a relation between integration constants $\pi$ and $V$ :

$$
\pi=\frac{\sqrt{3} c_{s} U_{1}}{3+\frac{\sqrt{3}}{\tau c_{s}}\left(\frac{L}{2}-\tau V\right)} .
$$

Similarly, at the top plate $(y=L / 2),\left.f_{4,7,8}\right|_{y=-L / 2}=$ $f_{4,7,8}^{\text {eq }}\left(\rho, \rho U_{2}, 0\right)$. Again, computing the difference $\left[f_{7}^{\text {neq }}-\right.$ $\left.f_{8}^{\text {neq }}\right]_{y=L / 2}$ in two ways as described above, we find 


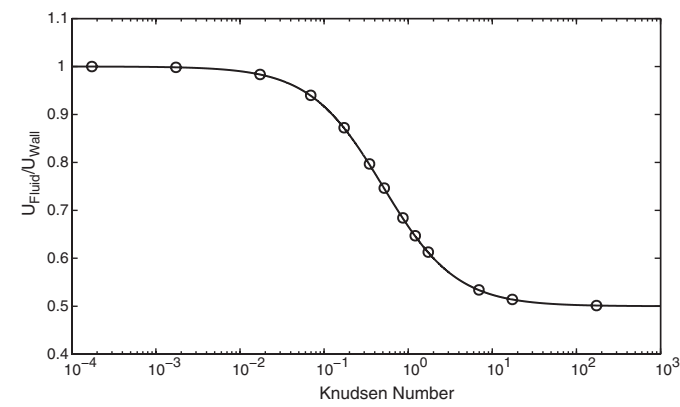

FIG. 3. Exact solution for the relative slip velocity (line) and the lattice Boltzmann simulation (symbol) as a function of Knudsen number.

$$
\pi=-\frac{\sqrt{3} c_{s} U_{2}}{3+\frac{\sqrt{3}}{\tau c_{s}}\left(\frac{L}{2}+\tau V\right)}
$$

Comparing (18) and (19), we find coefficients $V$ and $\pi$, and, making use of (13), we arrive at the result for the velocity profile (4), while the nonequilibrium shear stress $P_{x y}^{\text {neq }}=\rho \pi$ reads

$$
P_{x y}^{\mathrm{neq}}=-\frac{\nu \rho}{\Theta_{9}}\left(\frac{\Delta U}{L}\right) .
$$

Note that in the $D 2 Q 16$ model the result for the shear stress can be obtained by replacing $\Theta_{9}$ with $\Theta_{16}(6)$. Results for the shear stress are compared with the data of Willis [12] and DSMC simulation in Fig. 2. The limiting values of the effective shear viscosity, $\nu_{\text {eff }}=-P_{x y}^{\text {neq }} L(\Delta U \rho)^{-1}$, at the infinite Knudsen number are 0.723 for the $D 2 Q 9$ and 1.113 for the $D 2 Q 16$ models in units of $\sqrt{\frac{k_{B} T_{0}}{2 \pi m}} L$; the corresponding result for the Boltzmann-BGK equation is 1 [12].

The same method is used in order to evaluate the two remaining integration constants $A_{ \pm}$. Namely, we evaluate $f_{2}^{\text {neq }}$ (bottom plate) and $f_{4}^{\text {neq }}$ (top plate) in the two ways described above. After some algebra, we find the results for the nonequilibrium normal stress difference and the transversal energy flux

$$
N^{\text {neq }}=\rho\left(\frac{\Delta U}{L}\right)^{2} \frac{\tau \nu}{(1+2 \mathrm{Kn})^{2}}\left[2-e^{-1 / 2 \mathrm{Kn}} \cosh \left(\frac{y}{\mathrm{Kn} L}\right)\right],
$$

$$
\begin{aligned}
q_{y}^{\text {neq }}= & -\rho\left(\frac{\Delta U}{L}\right)^{2} \frac{\nu}{(1+2 \mathrm{Kn})^{2}}[2 y \\
& \left.-\mathrm{Kn} L e^{-1 / 2 \mathrm{Kn}} \sinh \left(\frac{y}{\mathrm{Kn} L}\right)\right]+2 U P_{x y}^{\text {neq }} .
\end{aligned}
$$

Expressions for the velocity $u_{x}(4)$, the shear stress $P_{x y}^{\text {neq }}$ (20), the normal stress difference $N^{\text {neq }}(21)$, and the energy flux $q_{y}^{\text {neq }}(22)$, when substituted into $f_{i}=f_{i}^{\text {eq }}+f_{i}^{\text {neq }}$ [see (2) and (16)], furnish the exact solution of Couette flow for the nonlinear $D 2 Q 9$ model. The same solution method is applicable to any member of the LB hierarchy although algebra becomes more involved. Solution of the $D 2 Q 16$ model leading to the result (5) will be presented in a detailed publication.

The normal stress difference (21) is a positive-definite function which is consistent with kinetic theory of gases. Importantly, the nonvanishing of $N^{\text {neq }}$ and $q_{y}^{\text {neq }}$ is the direct implication of the nonlinearity of the kinetic Eq. (1) [manifested by the $\Delta U^{2}$ dependence in (21) and (22)], and cannot be predicted on the basis of linearized kinetic theory [11,12]. Therefore, the DSMC method [10] was used in order to validate the solution in the nonlinear domain. The normal stress difference (21) is mapped onto DSMC data in Fig. 2 which qualitatively confirms the prediction. Finally, we validate the lattice Boltzmann method for the kinetic Eq. (1). It is evident in Fig. 3 that the simulated relative slip accurately reproduces the exact solution (4). The same holds for all other moments. Thus, applications of the LB method to microflows are by no means an "artifact of numerics" [9].

To conclude, our analytical results suggest that the hierarchy of lattice Boltzmann models is the way to approximate the kinetic theory. Without a denial of a body of LB simulations, it must be appreciated that only the exact solutions answer unambiguously the question of the physical validity of the method. Our results reveal that applications of LB methods to microflows should be based on LB models with larger velocity sets if one seeks a quantitative prediction, especially in the transient regime.

We acknowledge useful discussions of some of the results with G.D. Doolen, H. C. Öttinger, S. Succi, and V. Yakhot. Support by BFE Project No. 100862 (I. V. K.), by the ETH Project No. 0-20235-05 (N. I. P), by CCEMCH (I. V. K. and S. A.), and by NTU Office of Research (S. A.) is gratefully acknowledged.

*Corresponding author.

[1] A. Beskok and G.E. Karniadakis, Microflows: Fundamentals and Simulation (Springer, Berlin, 2001).

[2] S. Ansumali and I. V. Karlin, Phys. Rev. E 66, 026311 (2002).

[3] S. Succi, Phys. Rev. Lett. 89, 064502 (2002).

[4] S. Ansumali and I. V. Karlin, Phys. Rev. Lett. 95, 260605 (2005).

[5] V. Sofonea and R. Sekerka, J. Comput. Phys. 207, 639 (2005).

[6] S. Ansumali, I. V. Karlin, and H. C. Öttinger, Europhys. Lett. 63, 798 (2003).

[7] S. S. Chikatamarla and I. V. Karlin, Phys. Rev. Lett. 97, 190601 (2006).

[8] A. N. Gorban and I. V. Karlin, Invariant Manifolds for Physical and Chemical Kinetics (Springer, Berlin, 2005).

[9] L.-S. Luo, Phys. Rev. Lett. 92, 139401 (2004).

[10] G. A. Bird, Molecular Gas Dynamics and the Direct Simulation of Gas Flows (Clarendon Press, Oxford, 1994).

[11] C. Cercignani, Theory and Application of the Boltzmann Equation (Scottish Academic Press, Edinburgh, 1975).

[12] D. R. Willis, Phys. Fluids 5, 127 (1962). 